\title{
TADAKHKHUL AL-LUGHAH AL-INDUNISIYYAH FI KITABATI AL-LUGHAH AL- ARABIYYAH LADAY THALABATI AL-INDUNISIYYA
}

\author{
Ida Nur Chamidah
}

ida.chamidah@gmail.com

Pascasarjana UIN Maulana Malik Ibrahim Malang

\begin{abstract}
Language Interference to arabic language learners in Indonesia is a common phenomenon. Although the phenomenon is normal, but it is a problematic. Because each language has rules of each rule. It has been found forms of Indonesian interference into Arabic in the MAN Batu's students. The purpose of this study was to analyze the forms of interference Indonesian into Arabic in the students MAN Batu, uncover the causes of interference Languages Indonesisa into Arabic in the MAN Batu's students, and analyze solutions to the problem of interference Indonesian to Arabic in student writing MAN Batu. This study used a qualitative descriptive study, and the data collection with analysis of documentation and interviews. Source data is written documentation of students and teachers of Arabic. In this study, researcher found other forms of interference Indonesian into Arabic at the level of syntax, morphology and semantics. The causes interference Indonesian into Arabic in the MAN Batu's students that there is a cause intralinguistics and causes interlinguistics. The solution to the problem of interference is tahlil taqobuli and plenty of exercise in the writing.
\end{abstract}

Keywords: Tadakhkhul, al-Lughah al-Indunisiyyah, al-Lughah al-Arabiyyah, al-Asykal, ad-Dirasah at-Taqabuliyyah.

$$
\begin{aligned}
& \text { اللغة. ويرى البعض أن سبب بعض هذه الأخطاء يعود إلى }
\end{aligned}
$$

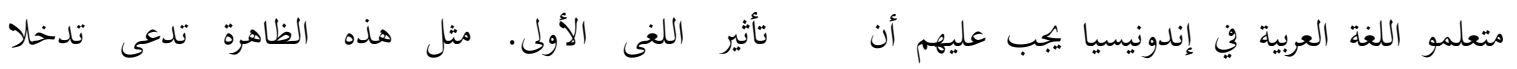

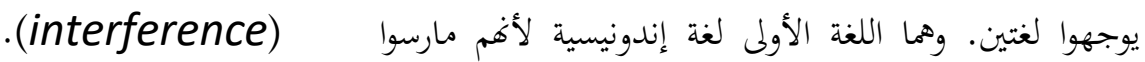

$$
\begin{aligned}
& \text { ويعني التدخل اللغوي المشكلات أو التدخلات }
\end{aligned}
$$

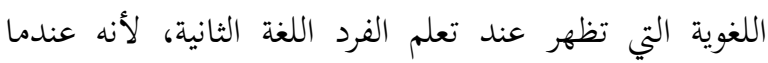

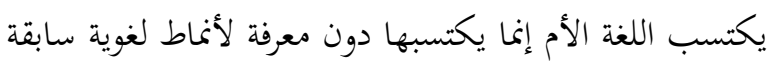

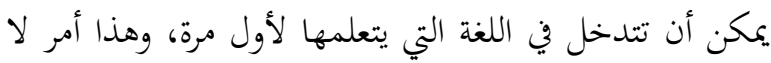

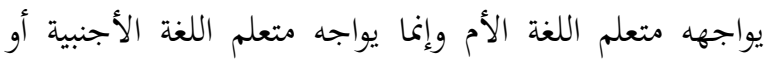

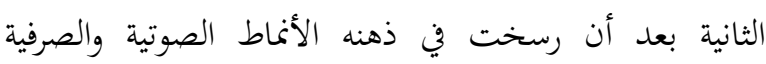

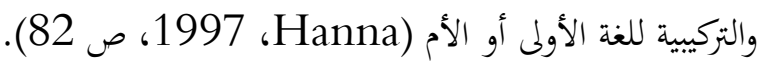

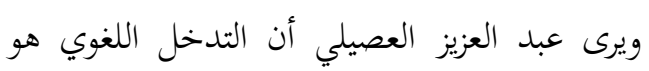

$$
\begin{aligned}
& \text { نقل المتعلم أنظمة لغته الأم وقواعدها إلى اللغة الثانية في كلام }
\end{aligned}
$$

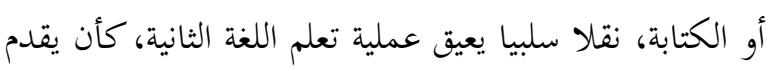


ولو تلك الظاهرة عادة ولكنها مشكلة. لأن اللغة لكلها قواعد. وقواعد اللغة الأولى بقواعد اللغة الأخرى مختلف. لذلك إذا كان تركيب اللغة العربية مركب بقواعد اللغة الإندونيسية فتلك اللغة غير صحيحة بل تلك اللغة فسادة.

ومن ذلك أن تدخل اللغة يجب عليه مبحوث لصحة اللغة. وأما الدراسة في تدخل اللغة قد عملها الباحثون قبلها وكثير من دراستهم في تدخل اللغة الجاوي في اللغة الإندونيسية، وبعض من دراستهم في تدخل اللغة الإندونيسية في اللغة العربية في الجامعة. إذن الدراسة في تدخل اللغة الإندونيسية في كتابة اللغة العربية في المدرسة الثانوية لما يعملها. ويهدف هذا البحث إلى الإجابة على عدد الأسئلة

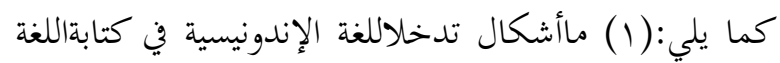

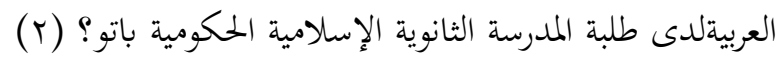
ما أسباب وقوع تدخل اللغة الإندونيسية فيكتابة اللغة العربية لدى طلبة المدرسة الثانوية الإسلامية الحكومية باتو؟ (r)ما الحلول لمشكلات تدخل اللغة الإندونيسية في كتابة اللغة العربية

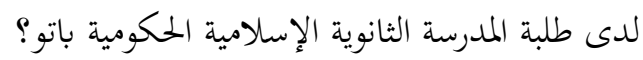

\section{منهجية البحث}

استخدمت الباحثة في هذا البحث المدخل الكيفي

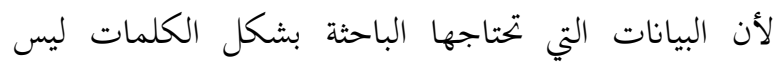

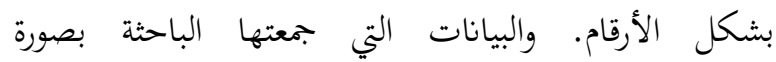
الكلمات.وأما منهج البحث الذي استخدمته الباحثة منهج وصفي تحليلي. وأما المصادر لهذا البحث هي: 1) وثائق كتابة

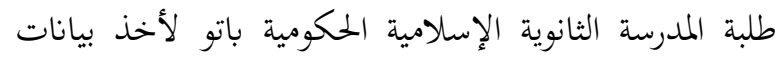
تدخل اللغة الإندونيسية في كتابة اللغة العربية، 2) مدرسة اللغة العربية في تلك المدرسة لأخذ بيانات أسباب وقوع تدخل اللغة

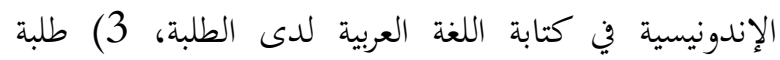
المدرسة الثانوية الإسلامية الحكومية باتو الذين في كتاباتم تدخل اللغة الإندونيسية.

وأما أسلوب جمع البيانات في هذا البحث هي تحليل الوثائق والمقابلة: 1) تحليل الوثائق، استخدمت الباحثة تحليل

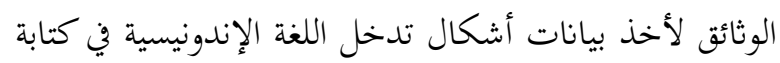

متعلم العربية الناطق بالإنجليزية الصفة على الموصوف أو المضاف إليه على المضاف متأثرا بلغته الأم (Dimyati)

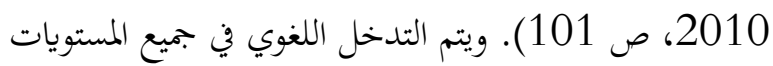
اللغوية: صوتيا، وصرفيا، ومفرداتيا، ونخويا، ودلاليا.

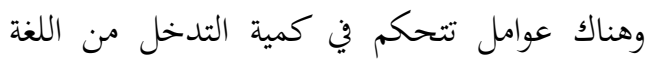
الأولى إلى اللغة الثانية. ومنها ما يلي (Dimyati.

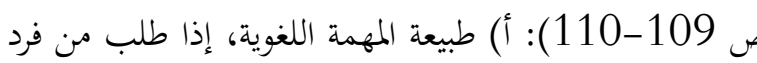

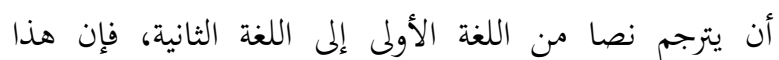
الموقف يفرض عليه التدخل من اللغة الأولى إلى اللغة الثانية،

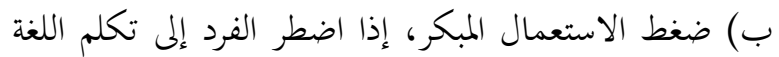

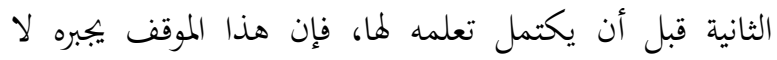

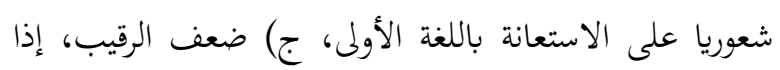

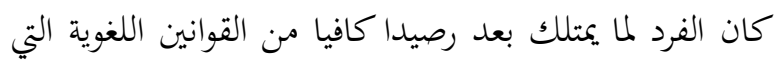

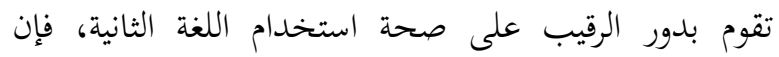
إنتاجه للغة الثانية سيتعرض للتدخل، د) إتقان اللغة الأولى واللغة الثانية، ذكرنا سابقا أنه كلما اتسع الفرق بين درجة

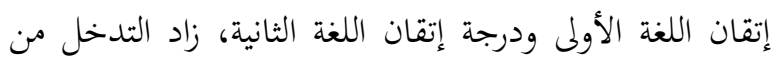

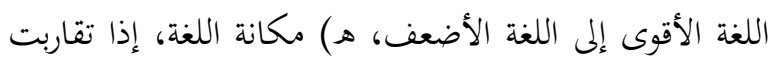

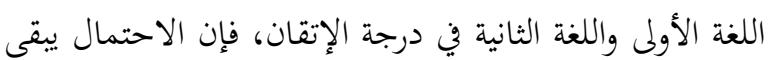

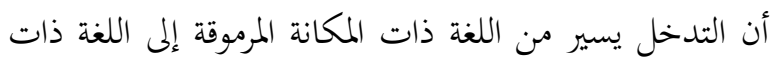

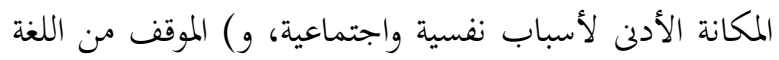

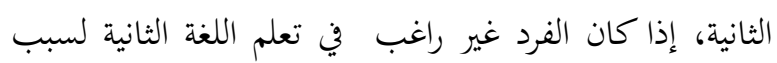
من الأسباب ولكنه مضطر إلى تعلمها لظروف معينة وكان في الوقت ذاته متمسكا باللغة الأولى ويخشى التخلي عنها لأنه يعتبرها رمزا لكرامته وثقافته وأصله وتراثه، في هذه الحالة تراه

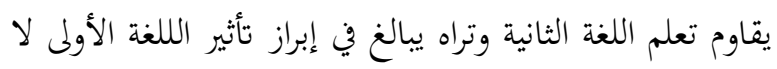
شعوريا. ومن وثائق كتابة اللغة العربية لدى طلبة المدرسة

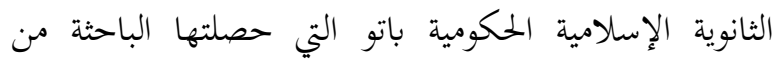
مدرسة اللغة العربية في تلك المدرسة في يوم السبت، بتاريخ 21 نوفيمبير 2015م، وقد وجدت فيها الباحثة أشكال تدخل اللغة الإندونيسية في كتابة اللغة العربية لديهم. 


\begin{tabular}{|c|c|}
\hline 1. & 1. لمتابة الطلبة بشكل \\
\hline 2. والكتابة في هذه الورقة & 2. والكتابة في هذه الورقة \\
\hline كتبها الطلبة ين & كتبها الطلبة في \\
\hline الفصل الحادي عشر & الفصل العاشر 4، \\
\hline قسم اللغة، & والكتابة في كل الورقة \\
\hline 3. ـ والكتابة في كل الورقة & كتبها طالبان وهناك \\
\hline كتبها طالبان وهناك & ثلاث طلبة. \\
\hline ثناث طلبة. & \\
\hline
\end{tabular}

تدخل اللغة هو من مصادر الأخطاء، لذلك لتعريف

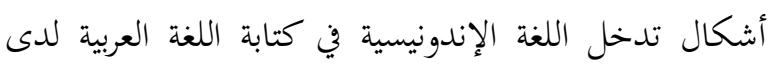
الطلبة قد قامت الباحثة بتحليل الأخطاء. وتحليل الأخطاء يمر

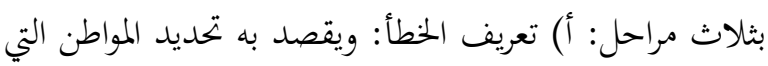
تنحرف فيها استجابات الطلاب عن مقاييس الاستخدام اللغوي الصحيح، ب) توصيف الخطأ: ويقصد به بيان أوجه

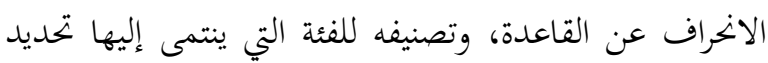

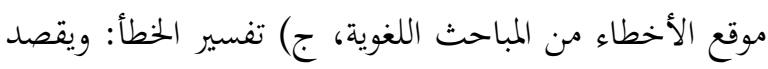

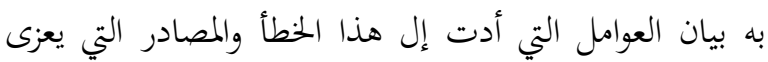
إليها.

ولتعريف الخطأ استخدمت الباحثة الكتب الذي

فيها قواعد اللغة العربية وفي تفسير الخطأ استخدمت الباحثة تحليل تقابلي بين اللغة الإندونيسية واللغة العربية لفحص أوجه

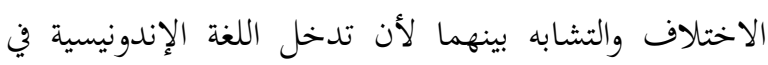

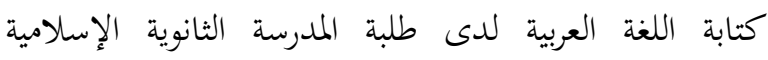
الحكومية باتو هو نقل طلبة المدرسة الثانوية الإسلامية

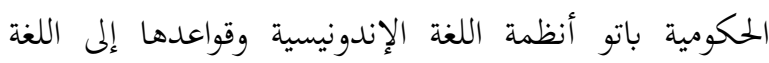
العبية في الكتابة. ومن ذلك لتحليل أشكال تدخل اللغة الإندونيسية في كتابة اللغة العربية لدى طلبة المدرسة الثانوية

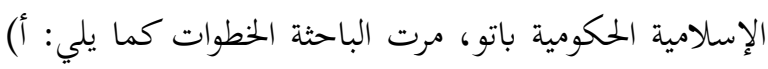

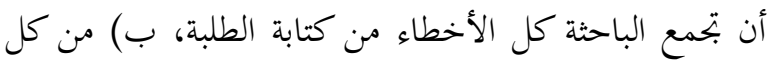
الأخطاء، تقوم الباحثة بتحليل الأشكال من الأخطاء، وتقوم

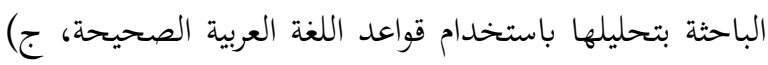
وبعد أن تعين الباحثة أشكال الأخطاء، تقوم الباحثة بمقارنة
اللغة العربية لدى طلبة المدرسة الثانوية الإسلامية الحكومية

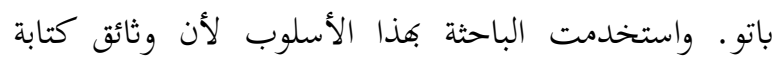
الطلبة في هذه المدرسة موجودة. وبالوثيقة لحفظ المحصول على

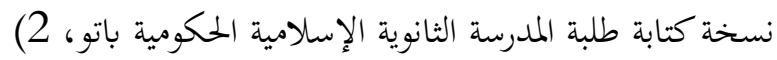
المقابلة، استخدمت الباحثة المقابلة لتحصيل بيانات أسباب لمابه وقوع تدخل اللغة الإندونيسية في كتابة اللغة العربية لدى الطلبة. وتقوم الباحثة بمقابلة موسعة مع مدرسة اللغة العربية في

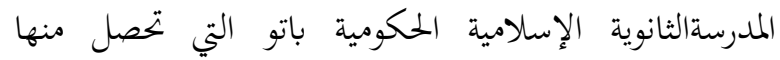

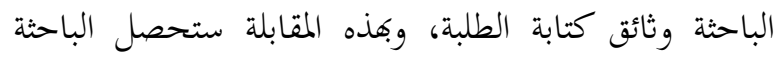

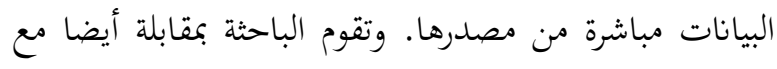

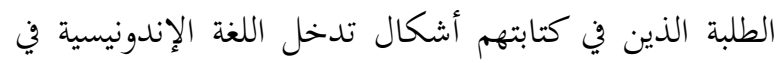

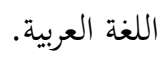

\section{عرض البيانات وتحليلها}

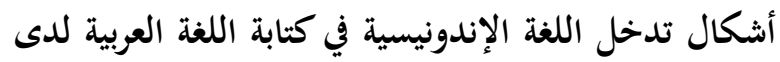
طلبة المدرسة الثانوية الإسلامية الحكومية باتو

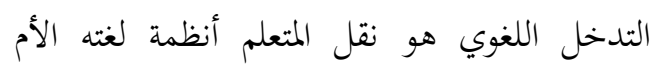

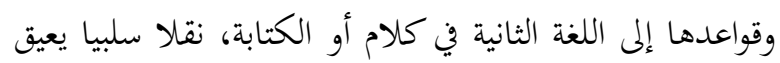

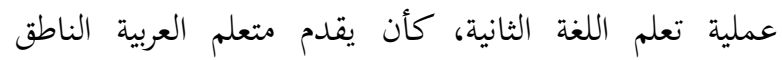

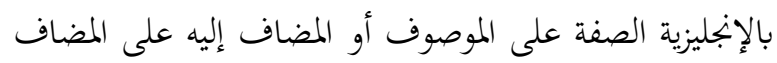

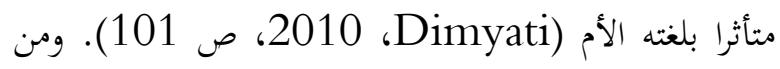
ذلك المقصود من تدخل اللغة الإندونيسية في كتابة اللغة العربية لدى طلبة المدرسة الثانوية الإسلامية الحكومية باتو هو الإسية نقل طلبة المدرسة الثانوية الإسلامية الحكومية باتو أنظمة اللغة الإندونيسية وقواعدها إلى اللغة العربية في الكتابة. ولتعريف أشكال تدخل اللغة الإندونيسية في كتابة اللغة العربية لدى طلبة المدرسة الثانوية الإسلامية الحكومية باتو، قامت الباحثة بتحليل الوثائق. والوثائق هي كتابات الإنية الطلبة التي قد حصلت الباحثة من مدرسة اللغة العربية في المدرسة الثانوية الإسلامية الحكومية باتو في يوم السبت، 21 نوفيمبير 2015. وتلك المدرسة هي استاذة ديان كوملا ساري. والوثائق نوعان، والخصائص منهما كما يلي: وليمير

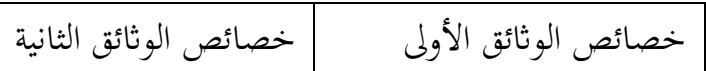


ونتائج تحليل الوثائق التي قد قامت بها الباحثة بالخطوات السابقة، قد وجدت ستة أشكال تدخل اللغة الإندونيسية في اللغة العربية في كتابة الطلبة. وستعرضها الباحثة مع كتابات الطلبة التي فيها أشكال تدخل اللغة الإندونيسية في

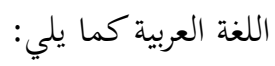

بين أشكال الأخطاء في كتابة الطلبة وأنظمة اللغة الإندونيسية وقواعدها، د) إذا وجدت متشابهة بين شكل الخطاء في كتابة الطلبة وأنظمة اللغة الإندونيسية وقواعدها فذلك الخطأ من

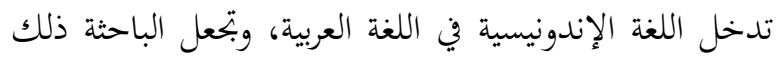

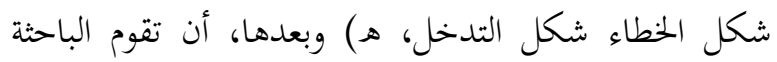
بتصنيف تدخل الإندونيسية في اللغة العربية من حيث أنواع

\begin{tabular}{|c|c|c|c|c|c|}
\hline الوثائق & اسم الطلبة & كتابة الطلبة & & أشكال التدخل & 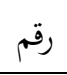 \\
\hline الأولى & عملي أزراء زين العابدين، & عائلتي كبير & بين & عدمام المطابقة & 1 \\
\hline الثانية & نور أنطان وحيودي، & المدرسة جميل & & & \\
\hline الأولى & فينا هميديا، & أنتما مدرسون & & & \\
\hline الأولى & 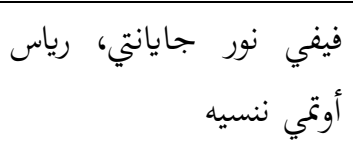 & هل أنتِ تَسْكُنُ مع أسرتكِ؟ & بين & الفعل وفامل المطابقة & 2 \\
\hline الثانية & فيكا أيو فاريرا سوكما فبريانتي، & الطلاب يذهب إلى المكتبة & & & \\
\hline الثانية & أيو إنداه ليستاري، & وقت الرحة يذهب إلى المقصف في & & & \\
\hline الثانية & نوفرال دفا، & أنا يذهبُ إلى المدرسة في كل يوم & & & \\
\hline
\end{tabular}




\begin{tabular}{|c|c|c|c|c|}
\hline ال الأولى & عملي أزراء زين العابدين، & ماذا تعمل أبك في هناك يا علي؟ & & \\
\hline ال الأولى & محمد مهارديكا، & وهل أبك وأمك يعمل في مالانج & & \\
\hline ال الأولى & خفالوه آنكون، & ما عندي أخت الصغير & الصفة والموصوف المطابقة بين & 3 \\
\hline ال الأولى & أروندا نور، & الأخت الكبير والاخ الصغير & & \\
\hline ال الأولى & تصنيا سومرا & 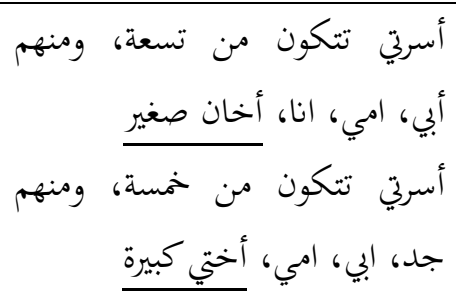 & & \\
\hline ال الأولى & عزيليا ايكا، & 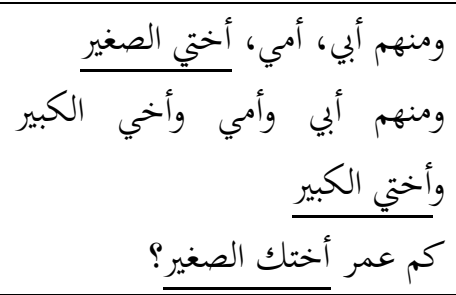 & & \\
\hline ال الأولى & علي أزراء زين العابدين، & ماذا تعمل أبك في هناك يا علي؟ & لبعض علامة الإعراب & 4 \\
\hline ال الأولى & محمد مهارديكا، & وهل أبك وأمك يعمل في مالانج & الخحمسة & \\
\hline الأولى & عزاميليا ايكا، & وكم أمرأخك الصغير؟ & & \\
\hline ال الأولى & 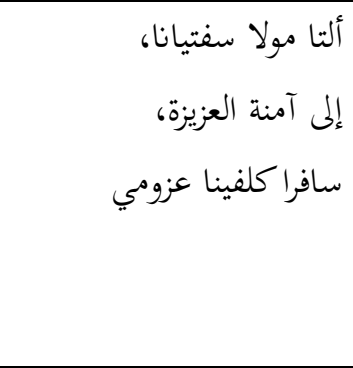 & 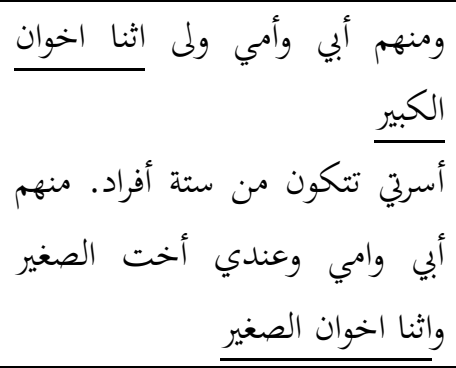 & لتئية الإسم العدد لثنا" & 5 \\
\hline الأولى & فيفي نور جايانتي، رياس & من اسم الأخ الصغير؟ اسمه دفن & استخدام كلمة "من" & 6 \\
\hline
\end{tabular}




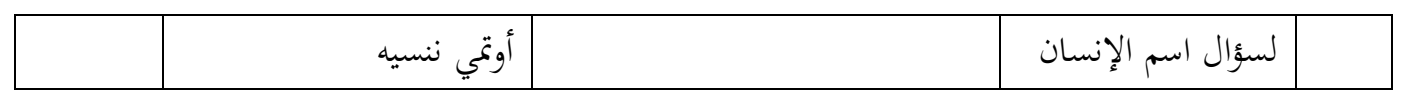

ومن أشكال تدخل اللغة الإندونيسية في اللغة العربية

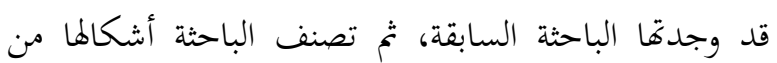
حيث أنواع التدخل اللغوي. وتصنيفها كما يلي:

\begin{tabular}{|c|c|c|}
\hline أنواع التدخل اللغوي & أشكال تدخل اللغة الإندونيسية في اللغة العربية & رقم \\
\hline \multirow[t]{4}{*}{ ت تدخل نغوي } & عدم المطابقة بين المبتدأ وخبره & 1 \\
\hline & عدم المطابقة بين الفعل وفاعله & 2 \\
\hline & عدم المطابقة بين الصفة والموصوف & 3 \\
\hline & عدم علامة الإعراب لبعض الأسماء الخمسة & 4 \\
\hline تدخل صري & ذكر العدد "إثنا" لتثنية الإسم & 5 \\
\hline تدخل دلالي & استخدام كلمة "من" لسؤال اسم الإنسان & 6 \\
\hline
\end{tabular}

ترتيب الكلمات الخاص باللغة الثانية، أي يتدخل نخو

وأما تحليل أشكال تدخل اللغة الإندونيسية في اللغة

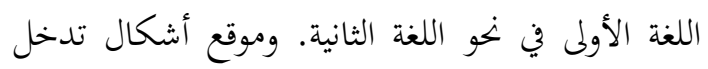

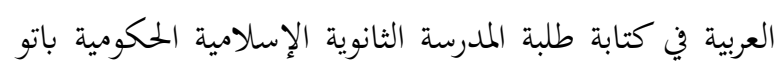

نغو اللغة الإندونيسية في نخو اللغة العربية في كتابة الطلبة

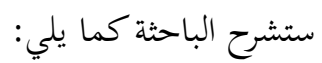

ووصفها كما يلي:

أ) أشكال التدخل النحوي، في التدخل النحوي، يتدخل

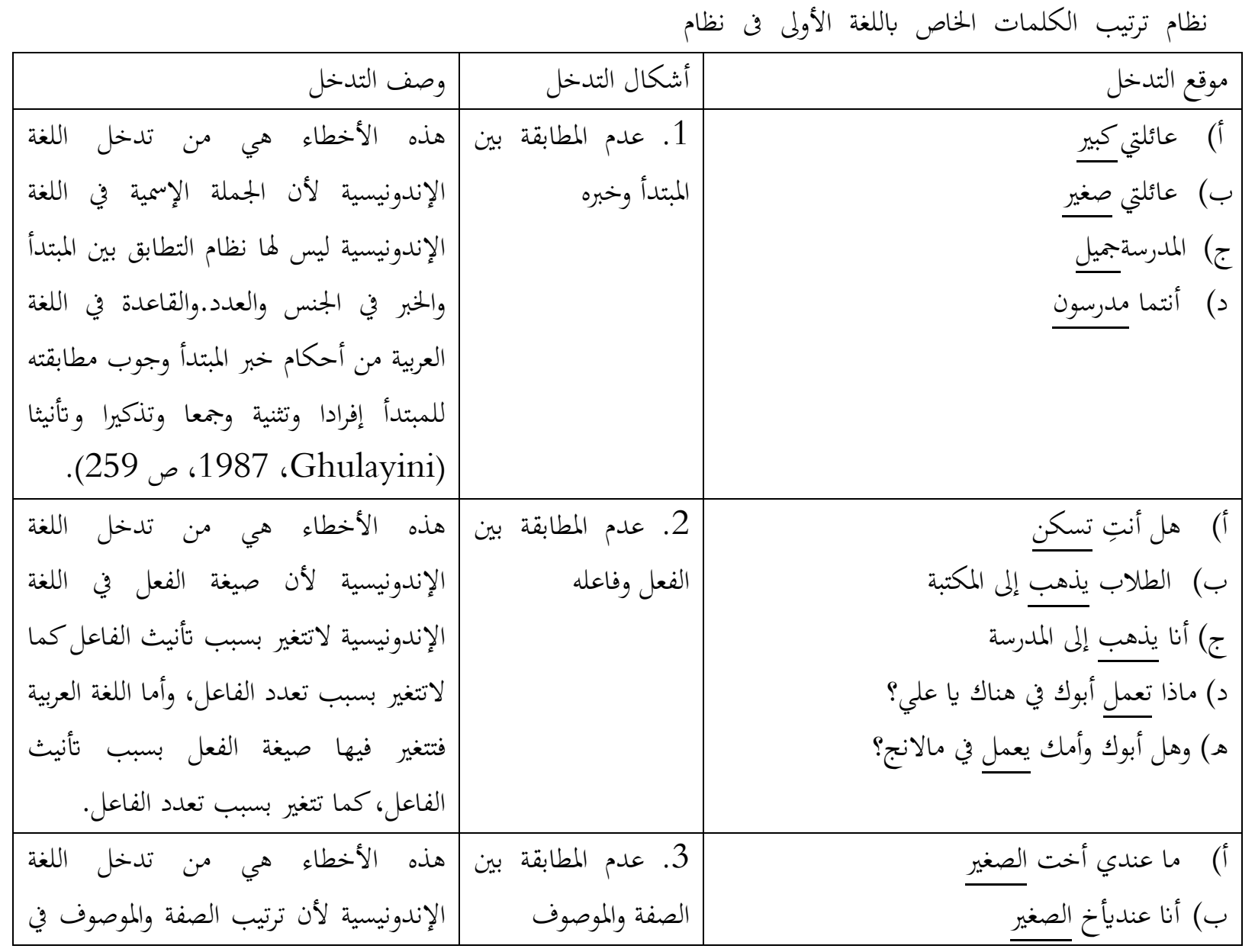




\begin{tabular}{|c|c|c|}
\hline اللعيين الوصوف الإندونيسية لا تتغير فيها الصفة بتغير والعدده ونوعه، والقاعدة في & & 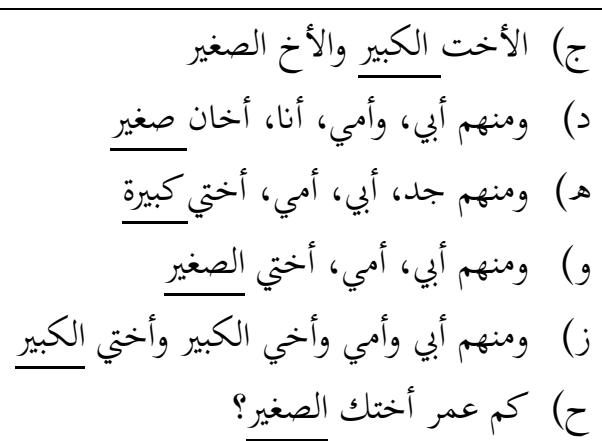 \\
\hline 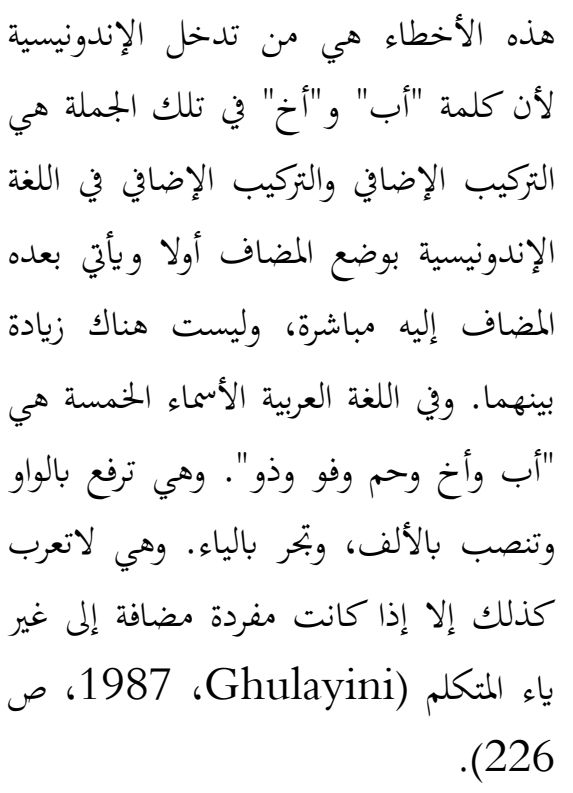 & الإعراب لبعض الأسماء & 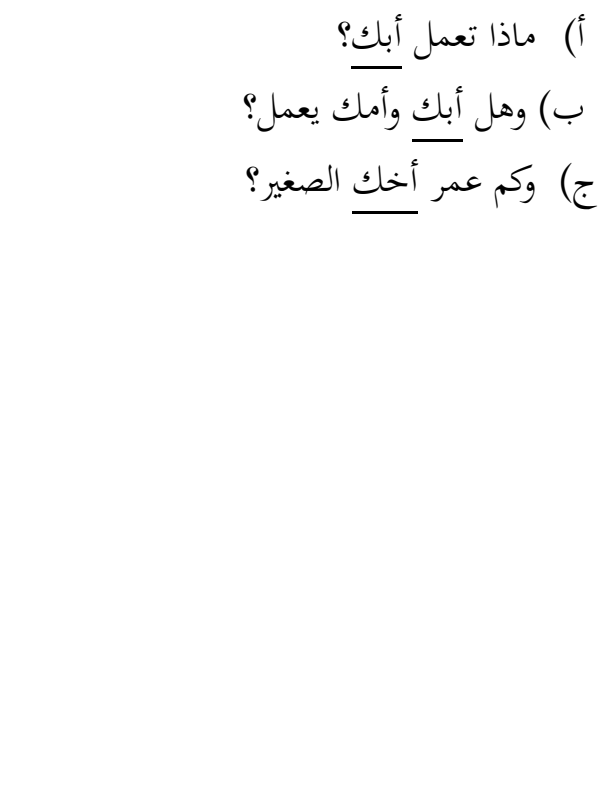 \\
\hline
\end{tabular}

اللواحق (suffixes) ونظام الدواخل (infixes)

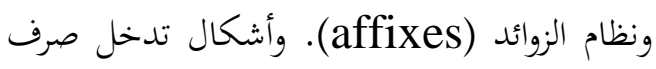
ب) أشكال الثدخل الصرفي، التدخل الصرفي يعني أن اللغة الإندونيسية في صرف اللغة العربية في كتابة يتدخل صرف اللغة الأولى في صرف اللغة الثانية.

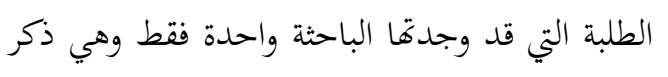
مثال ذلك جمع الاسم وتثنيته وتأنيثه وتعريفه وتصغيره

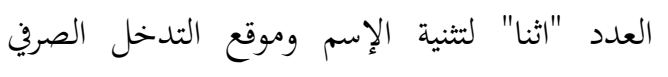
وتحويل الفعل من ماض إلى مضارع إلى أمر ونظام

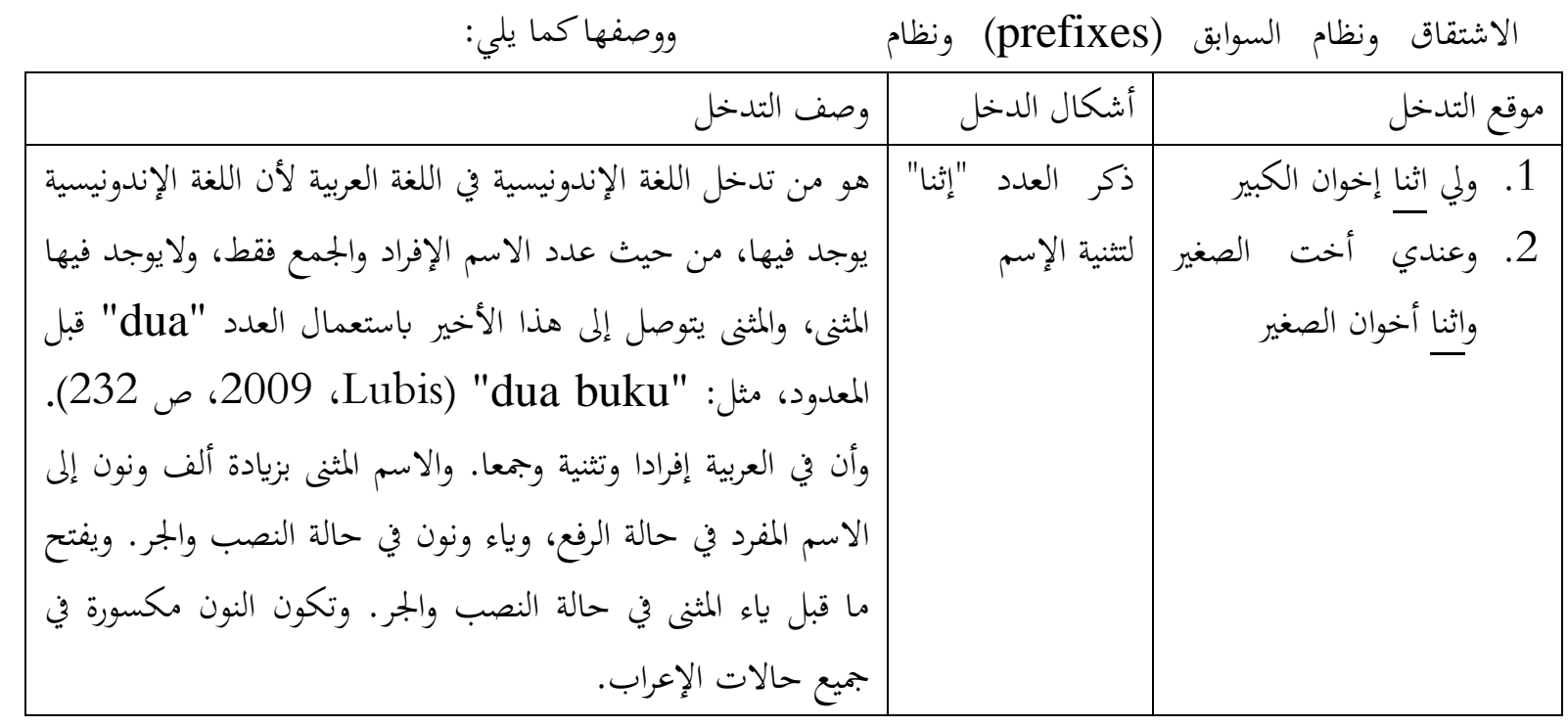


لإقامة المقابلة مع أستاذة ديان قد سألتها الباحثة

عن أسباب وقوع ظاهرة تدخل اللغة الإندونيسية في اللغة العربية مباشرة في تاريخ 11 مارس 2016 يوم الجمعابة. والسؤال الرئيسي من الأسئلة التي قدمتها الباحثة هو ما أسباب وقوع ظاهرة تدخل اللغة الإندونيسية في كتابة اللغة العربية لدى

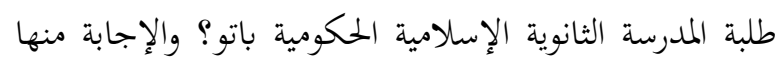

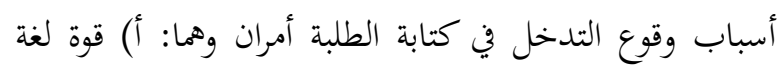
الأم لطلبة أي اللغة الإندونيسية، ب) نسيان من الطلبة.

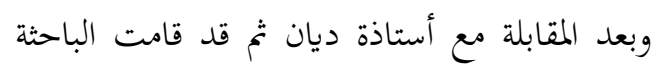
مقابلة مع الطلبة التي في كتابتهم تدخل اللغة الإندونيسية في

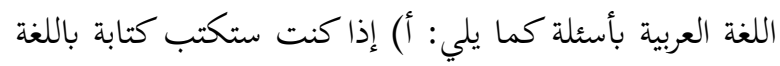

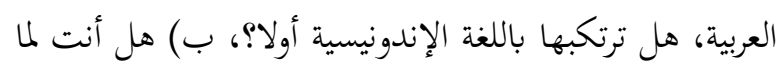

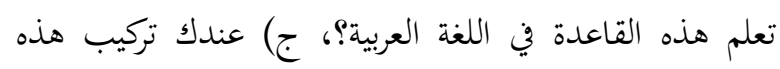

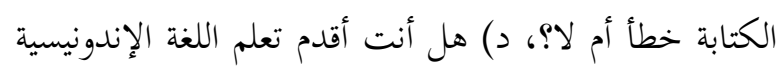

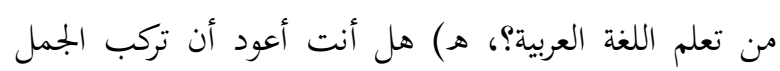

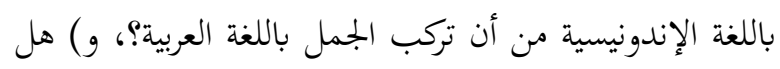

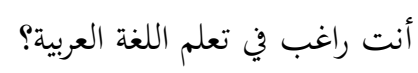

ومن نتائج المقابلة مع مدرسة اللغة العربية والطلبة

الذين في كتاباتم تدخل اللغة الإندونيسية في اللغة العربية، معروف أن أسباب وقوع ظاهرة تدخل اللغة الإندونيسية في كتابة اللغة العربية لدى طلبة المدرسة الثانوية الإسلامية الحكومية باتو فهي كما يلي: أ) كون عملية الترجمة للطلبة حين التحلية يكتبون، من نتائج المقالبة في سؤال رقم (أ) مع ألتا مولا

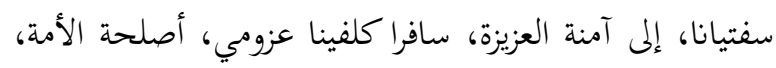

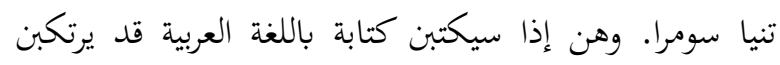
باللغة الإندونيسية أولا، ب) لما يكتمل تعلم اللغة العربية

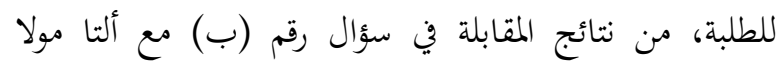

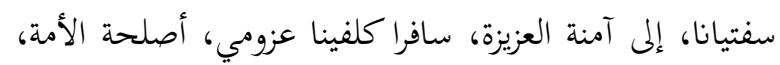

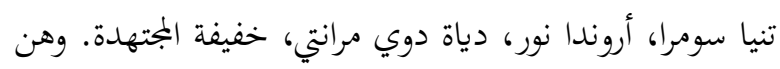

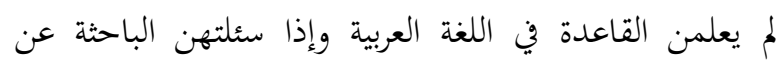
القاعدة في الكتابة التي فيها تدخل اللغة الإندونيسية وهن لايعلمن، ج) ضعف الرقيب على صحة استخدام اللغة

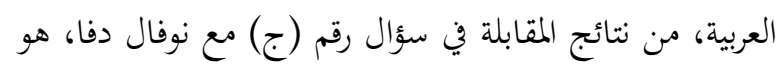

ج) أشكال التدخل الدلالي، التدخل الدلالي يعني تتدخل

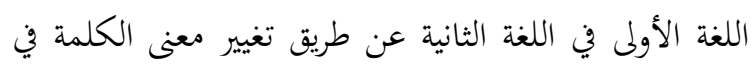

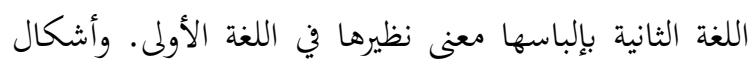
تدخل دلال اللغة الإندونيسية في دلال اللغة العربية في كتابة الطلبة التي قد وجدهّا الباحثة هي استخدام "من" لسؤال

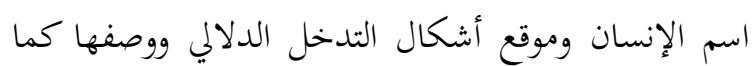

\begin{tabular}{|c|c|c|}
\hline وصف التدخل & أشكال & موقع \\
\hline 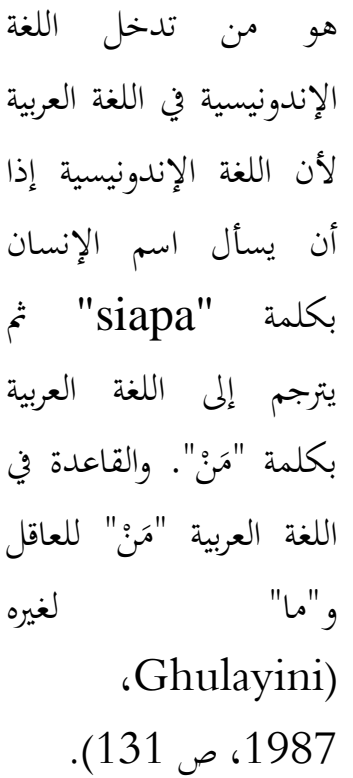 & "من" استخدام اسؤال & الصغير؟ اسم الأخ \\
\hline
\end{tabular}

ولا بتحد الباحثة تدخل صوتي في تلك الكتابة لأنه في الكتابة. ولابحد تدخل مفرداتي وهذا يعني لايكتب الطلبة مفردات اللغة الإندونيسية في كتابتهم. ولاتحد تدخل حركي وتدخل ثقافي لأن الكتابة السابقة بسيطة.

أسباب وقوع تدخل اللغة الإندونيسية في كتابة اللغة العربية

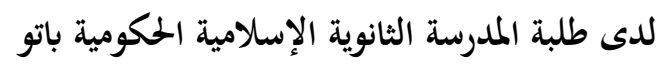

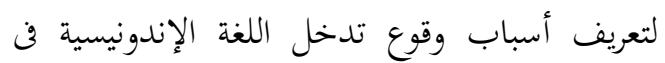
كتابة اللغة العربية لدى طلبة المدرسة الثانوية الإسلامية الحكومية باتو قد قامت الباحثة بمقابلة مع مدرسة اللغة العربية التي قد حصلت منها الباحثة وثائق كتابة الطلبة، واسمها أستاذة ديان كوملاساري والمقابلة مع الطلبة الذين في كتابتهم تدخل اللغة الإندونيسية في كتابة اللغة العربية. 
الحلول لمشكلات تدخل اللغة الإندونيسية في كتابة اللغة

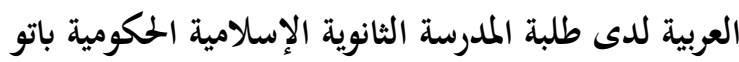
وبعد أن تكشف الباحثة أسباب وقوع تدخل اللغة الإندونيسية في كتابة اللغة العربية لدى طلبة المدرسة الثانوية الإسلامية الحكومية باتو في هذا الباب ستقدم الباحثة الحلول

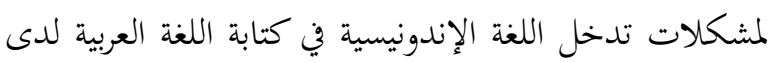

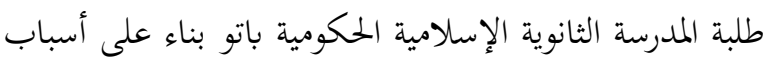

$$
\text { وقوع التدخل اللغوي في هذه المدرسة. }
$$

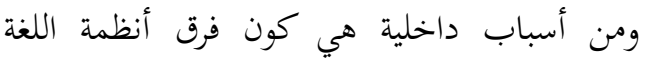

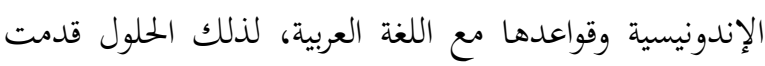

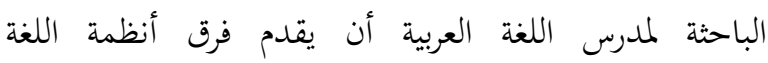
الإندونيسية وقواعدها مع اللغة العربية إذا يقدم المادة في تعليم

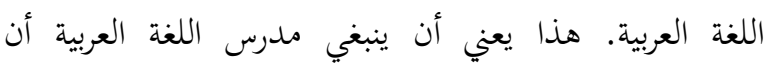
يستخدم تحليل تقابلي أو التقابل اللغوي في تعليم اللغة العربية.

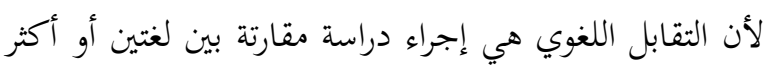

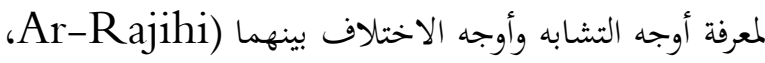

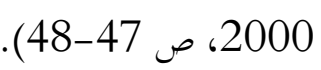

ولكن ظهر ابتحاه تحليل الأخطاء في أواخر الستينات

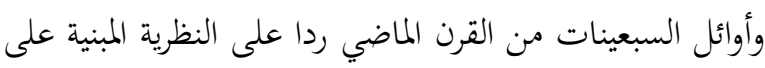

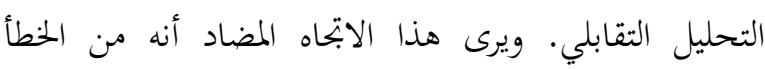

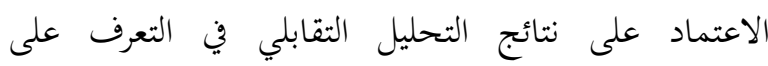

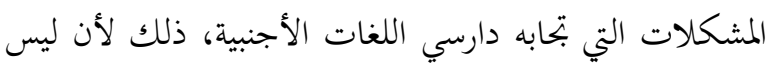
كل ما يتوقع حدوثه نظريا بواسطة التحليل التقابلي يقع

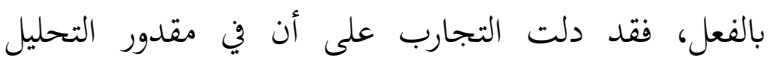
التقابلي أن يتنبأ 50 إلى 60\% فئس من من الأخطاء الحقيقية.

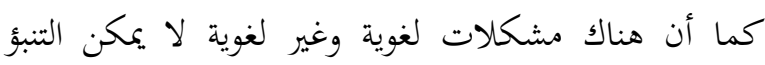

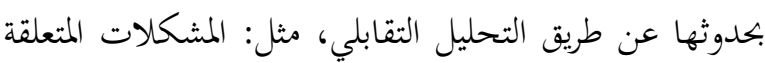

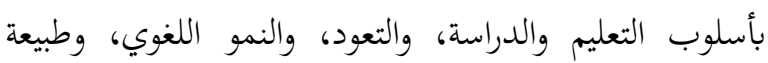
اللغة المدروسة، وهدف الدارسين وسننهم (Nashruddin، 2005، ص 178).

ومن ذلك، لو تحليل تقابلي هو الحلول لمشكلات

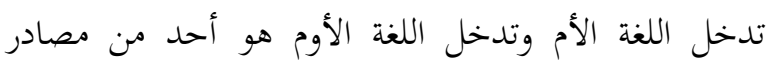

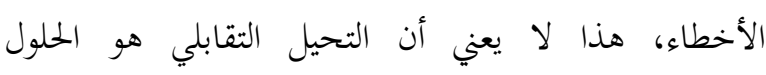

كتب "أنا يذهب" ولكن عنده هذا خطاء والصحيحة "أنا

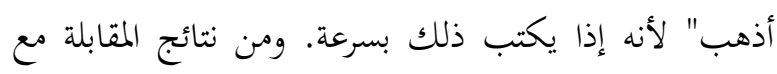

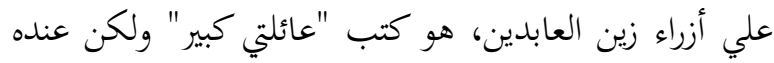
هذا خطاء والصحيحة "عائلتي كبيرة"، لأنه حين يكتب بدئ بدون استخدام دور الرقيب على صحة استخدام اللغة العربية، د) قوة اللغة الإندونيسية للطلبة، من نتائج المقابلة في سؤال رقم (د) مع ربيعة النزاء، يونيار ايلدي، وكثير من طلبلة إتقان اللغة لإنة

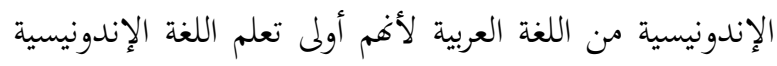

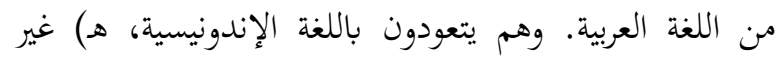

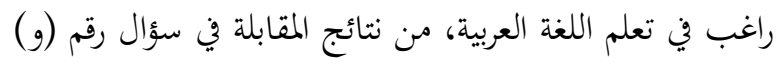

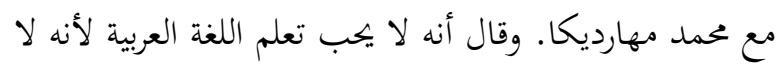
يحب أن يحفظ وعنده أن تعلم اللغة العربية كثر الحفظ.

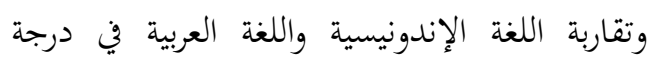
الإتقان ليست من أسباب وقوع ظاهرة تدخل اللغة ولغة ولنة الإندونيسية في اللغة العربية في كتابة طلبة المدرسة الثانوية الإسلامية الحكومية باتو لأن كل الطلبة يعودون أن أن يركبوا

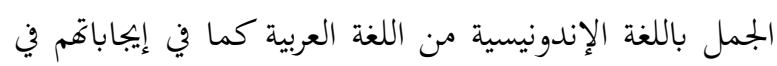
سؤال في رقم (ه). ومن العوامل الستة تتحكم في كمية التدخل من اللغة الأولى إلى اللغة الثانية في الإطار النظاري، أسباب وقوع تدخل

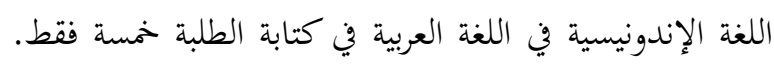
ولأن تلك الخمسة هي أسباب من خارج اللغة. لذلك تلك الإنك الخمسة هي أسباب خارجية وقوع تدخل اللغة الإندونيسية في اللغة العربية في كتابة الطلبة. ومن نتائج تحليل أشكال التدخل اللغة الإندونيسية

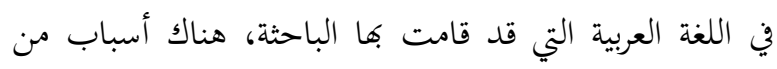

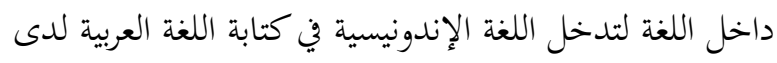
الطلبة فهي كون فرق أنظمة اللغة الإندونيسية وقواعدها مع الإنسية

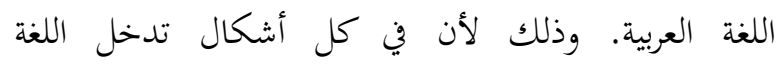
الإندونيسية في كتابة اللغة العربية لدى الطلبة هناك فرق أنظمة اللغة الإندونيسية وقواعدها مع اللغة العربية. 
لسؤال اسم الإنسان. (2) أسباب وقوع تدخل اللغة الإندونسيسة في كتابة اللغة العربية لدى طلبة المدرسة الثانوية

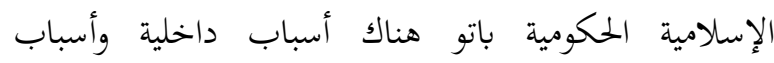
خارجية. أسباب داخلية هي فرق أنظمة اللغة الإندونيسية وقواعدها مع اللغة العربية. وأسباب خارجية هي كون عملية الترجمة للطلبة حين يكتبون، وملا يكتمل تعلم اللغة العربية للطلبة، وضعف الرقيب على صحة استخدام اللغة العربية، وقوة اللغة الإندونيسية للطلبة، وغير راغب في في تعلم اللغة العربية. (3) الحلول لمشكلات تدخل اللغة الإندونيسية في كتابة اللغة العربية لدى طلبة المدرسة الثانوية الإسلامية

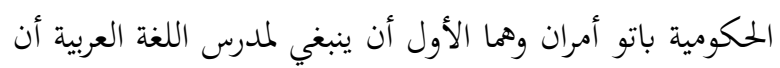

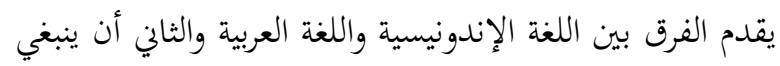
لمدرس اللغة العربية أن يعطي تدريبات الكتابة كثيرا. وانطلاقا من نتائج البحث تقدم الباحثة التوصيات كما يلي:(1) أن تهتم مدرسة اللغة العربية في المدرسة الثانوية

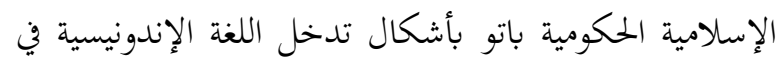

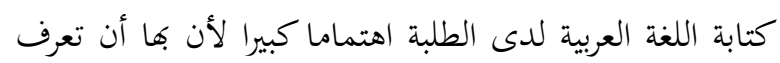

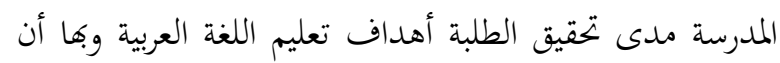

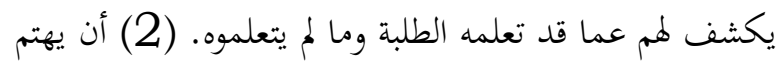

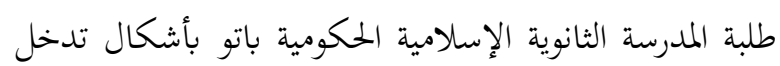

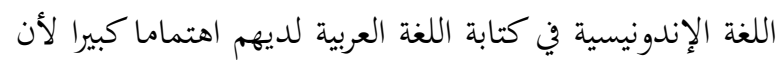

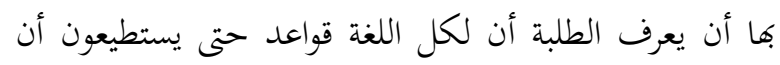
ينقصوا تدخل اللغة في كتابتهم.
لمشكلات كل الأخطاء لأن هناك مصادر الأخطاء أخرى غير

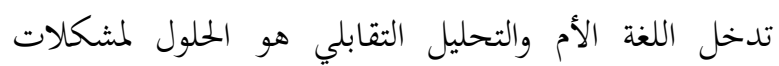

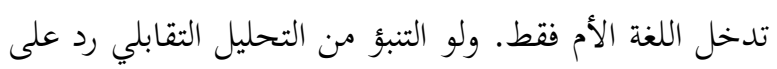

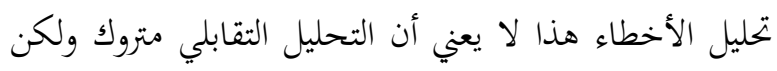

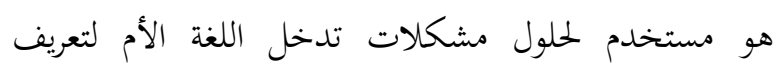
الإختلاف والتشابه بين اللغة الأم واللغة الهدف. وأسباب خارجية هي كون عملية الترجمة للطلبة حين

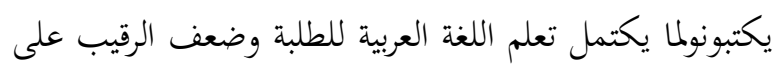

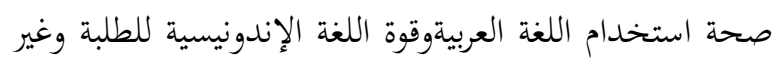

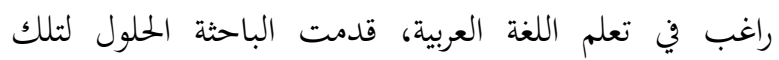
المشكلات لمدرس اللغة العربية أن يعطي تدريبات الكتابة كثيرا لطلبة. خاتمة وبعد أن قامت الباحثة بتحليل أشكال تدخل اللغة الإندونيسية في كتابة اللغة العربية لدى طلبة المدرسة الثانوية

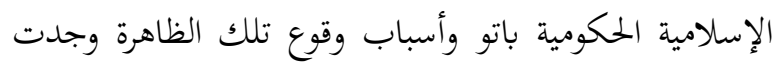
الباحثة ما يلي:(1) أشكال تدخلاللغة الإندونيسية في كتابة

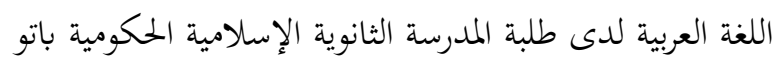

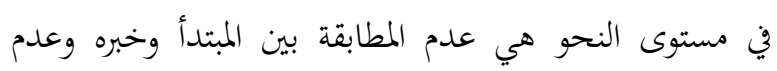

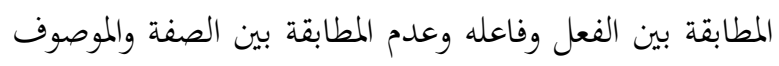

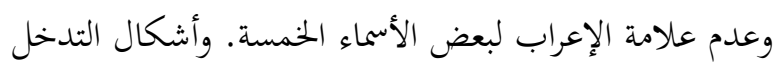

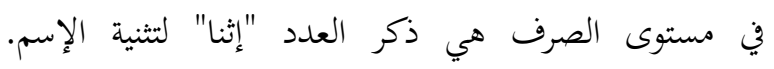
وأشكال التدخل في مستوى الدلال هي استخدام كلمة "من" دمر العند

\section{والمراجع}

Ar-Rajihi, Abduh. (2000). Ilmu al-Lughah al-Tathbiqi wa Ta'lim al-Lughah al-Arabiyyah. alIskandariyyah, Dar al-Ma'rifat al-Jami'iyyah.

Brown, H. Douglas. (1994). Principles of Language Learning and Teaching, New Jersey: Prentice Hall Regents.

Dimyati, Muhammad Afifuddin. (2010). Muhadlarah fi Ilm al-Lughah al-Ijtima'iyyah. Surabaya: Dar al-Ulum al-Lughawiyyah. 
Ghulayini, Muthofa. (1987). Jami' ad-Durus al-Arabiyyah. Beirut: al-Maktabah al-'Ashriyyah.

Lubis, Turkis. (2009, December). At-Taqaabul Baina Al-Lughatain Al-Arabiyyah Wa AlIndunisiyyah. Lingua Journal, Ed.

Nashruddin. (2005). Al-Usus al-Lughawiyyah li Bina'i Manhaj Ta'lim al-Lughah al-Arabiyyah li alNathiqina bi Ghairiha. Duna al-Mathba'.

Sami A. Hanna \& Others. (1997). Dictionary of Modern Linguistics: English-Arabic. Beirut: Libraire du Liban. 